\title{
Zur Kataloghaftung des Auktionshauses
}

LG Köln Urteil vom 5.10.2017 - 200 59/16

\section{ECLI:}

\section{ECLI:DE:LGK:2017:1005.20059.16.00}

\section{[1] Tatbestand:}

[2] Der Kläger begehrt von der Beklagten Schadensersatz wegen angeblich fehlerhafter Bewertung und Bewerbung eines Objekts des Klägers, das durch die Beklagte versteigert wurde.

[3] Der Kläger und sein Bruder, Herr Ferdinand Graf von G, erbten von ihrem Vater und ihrem Onkel u.a. einen antiken chinesischen Kendi. Dieser Kendi ist in dem Fachbuch von Lunsingh Scheurleer, Chinesisches und japanisches Porzellan in europäischen Fassungen, Braunschweig, 1980, auf S. 192, Abb. 41, abgebildet und wird dort besprochen. U.a. wird dort erwähnt, dass der Kendi im Besitz des G1 war und sich auf Schloß G befand.

[4] Am 23.10.2014 erteilte der Bruder des Klägers der Beklagten einen Auktionsauftrag, indem der Kendi nach Alter und Periode wie folgt beschrieben wurde: „China, Blau-weißes Kendi mit vergoldeter Silbermontierung, Wanli-Periode (1572-1620)“. Zum Zeitpunkt der Einlieferung des Kendi bei der Beklagten fehlten diesem sowohl der in dem Fachbuch noch abgebildete silberne Deckel als auch der dort noch zu sehende silberne Ausgießer.

[5] Bei der Beklagten handelt es sich um ein Auktionshaus, das ständig Antiquitäten und Kunstgegenstände zur Versteigerung annimmt. Zweimal im Jahr führt sie Spezialauktionen für asiatische Kunst durch. Der Kläger und sein Bruder waren bereits früher Kunden der Beklagten. Vor acht bis zehn Jahren hatten sie einmal an die Beklagte die Bitte gestellt, bei einer Versteigerung die Provenienz nicht anzugeben.

[6] Die Beklagte beschrieb den streitgegenständlichen Kendi unter Losnummer 195 in ihrem für die Auktion 1044 „Asiatische Kunst" herausgegebenen Katalog mit einer ganzseitigen Abbildung. In der englischen Fassung der Beschreibung befand sich unter der Überschrift "Literature" der Hinweis, dass der Kendi mit dem auf S. 192, Abb. 41, in dem Fachbuch von Lunsingh Scheurleer gezeigten Kendi vergleich bar ist ("compare an almost identical kendi"). Für die Beschreibung und die Abbildung wird auf die Anlage B 1, Bl. 62 d. A. verwiesen. Der Schätzpreis wurde mit 3.000-4.000 EUR beziffert. Die Beklagte bewarb den Kendi darüber hinaus online und in ihrem Katalog für „Europäische Kunst“. Die Provenienz aus dem gräflichen Besitz von G wurde nicht erwähnt.
[7] Die Beklagte versteigerte den streitgegenständlichen Kendi in der Auktion 1044 für asiatische Kunst am 5.12.2014. Es hatten mehrere Telefonbieter Gebote auf den Kendi abgegeben. Zudem boten mehrere im Saal anwesende Bieter persönlich mit. Der Zuschlag wurde bei 75.000 EUR erteilt.

[8] Wenige Monate später wurde der Kendi von dem Auktionshaus Sotheby's in einem Versteigerungskatalog mit einem Schätzpreis von $200.000,00$ bis $300.000,00$ GBP beworben. Für den Condition Report wird auf die Anlage K7, BI. 27 der Akte, und für eine weitere Beschreibung durch Sotheby's wird auf die Anlage K11, Bl. 40 ff. der Akte, verwiesen. Im Rahmen der Auktion durch Sotheby's am 8.7.2015 wurde der Zuschlag auf ein einziges abgegebenes Gebot bei 200.000 GBP erteilt (zum Zeitpunkt der Klage umgerechnet 277.619,30 Euro).

[9] Der Kläger behauptet, dass sich der tatsächliche Marktwert des Kendi mindestens auf 200.000 GBP belaufe. Die Beklagte habe den Wert des Verkaufsgegenstandes verkannt und keine geeigneten Maßnahmen ergriffen, um diesen wertentsprechend zu veräußern. Der zu niedrig eingeschätzte Wert, die unvollständigen Angaben im Auktionskatalog über die Provenienz des Gegenstandes und die falsche Angabe des Gegenstands als nur ähnliches Exemplar, anstatt als das im Fachbuch abgebildete Exemplar, hätten zu dem wesentlich niedrigeren Versteigerungserlös geführt, als er bei der später stattgefundenen Auktion von Sotheby's erzielt wurde. Der Anlass für die frühere Bitte um Nichtangabe der Provenienz bestehe nicht mehr. Dass sei der Beklagten auch bekannt gewesen. Die Beklagte habe keine ausreichenden Nachforschungen über den Wert des Kendi vorgenommen. Sie habe die Auktion des Kendi zurücknehmen müssen, als sie bemerkte, dass er mit dem in der Fachliteratur besprochenen Kendi identisch ist.

[10] Der Kläger ist der Ansicht, dass ihm die Beklagte daher zur Zahlung von Schadensersatz verpflichtet sei. Der kausale Schaden in der Höhe des Differenzbetrages zwischen dem Versteigerungserlös bei Sotheby's abzüglich 9\% Provision und des von der Beklagten ausgekehrten Erlöses sei ihm von der Beklagten als entgangener Gewinn zu ersetzen.

[11] Ursprünglich beantragte der Kläger,

[12] 1. die Beklagte zu verurteilen, an den Kläger 184.383,56 EUR zuzüglich Zinsen in Höhe von 5 Prozentpunkten seit dem 29.11.2015 zu zahlen;

[13] 2. die Beklagte zu verurteilen, an den Kläger weitere 3.006,42 EUR zuzüglich Zinsen in Höhe von 5 Prozentpunkten seit dem 15.12.2015 zu zahlen. 
[14] Nunmehr beantragt der Kläger anstelle des Hauptantrags, wie zunächst nur hilfsweise beantragt,

[15] 1. die Beklagte zu verurteilen, an die Erbengemeinschaft nach $\mathrm{G} 3$ und G4, bestehend aus dem Kläger und seinem Bruder G2, 184.383,56 EUR zuzüglich Zinsen in Höhe von 5 Prozentpunkten über dem Basiszinssatz seit dem 29.11.2015 zu zahlen;

[16] 2. die Beklagte zu verurteilen, an die Erbengemeinschaft nach G3 und G4, bestehend aus dem Kläger und seinem Bruder G2, weitere 3.006,42 EUR zuzüglich Zinsen in Höhe von 5 Prozentpunkten seit dem 15.12.2015 zu zahlen.

[17] Die Beklagte beantragt,

[18] die Klage abzuweisen.

[19] Die Beklagte behauptet, ihr Schätzpreis beruhe auf einer Abstimmung zwischen dem Bruder des Klägers und der Beklagten. Sie habe nach Veröffentlichung des Auktionskatalogs erkannt, dass der streitgegenständliche Kendi selber in dem Buch von Lunsingh Scheurleer abgebildet ist. Die Angabe sei dann in einer Korrigenda/Addenda-Liste korrigiert worden. Zudem sei in der streitgegenständlichen Auktion darauf hingewiesen worden, dass der Kendi in dem Buch von Lunsingh Scheurleer abgebildet ist.

[20] Die Beklagte ist der Ansicht, dass sie davon ausgehen durfte, dass die nicht widerrufene Anweisung zur Nichtangabe der Provenienz auch für die Versteigerung des Kendi gelte. Ihr seien im Zusammenhang mit der Versteigerung des Kendi keine von ihr verschuldeten Pflichtverletzungen vorzuwerfen, die zu einem kausalen Schaden geführt hätten.

[21] Das Gericht hat in der mündlichen Verhandlung am 29.6.2017 Beweis erhoben durch Vernehmung des Zeugen Dr. $\mathrm{H}$ und durch Einholung eines Sachverständigengutachtens von Dr. Q. Wegen des Ergebnisses der Beweisaufnahme wird auf das Sitzungsprotokoll vom 29.6.2017 (Bl. 223 ff. d. A.) Bezug genommen.

[22] Wegen des weiteren Sach- und Streitstandes wird auf die gewechselten Schriftsätze der Parteien nebst Anlagen sowie auf das Protokoll der mündlichen Verhandlung vom 29.6.2017 Bezug genommen.

\section{[23] Entscheidungsgründe:}

[24] Die zulässige Klage ist unbegründet.

\section{[25] I.}

[26] Der Kläger hat gegen die Beklagte nach der einzig in Betracht kommenden Anspruchsgrundlage gemäß §§ 280 I, III, 281, 248 I, 252 BGB iVm § 384 HGB keinen Anspruch auf Schadensersatz.
[27] Den Anspruchsteller trifft nach den allgemeinen zivilprozessualen Regeln die Darlegungs- und Beweislast hinsichtlich der anspruchsbegründenden Tatsachen, nämlich im konkreten Fall einer vertraglichen Pflichtverletzung, die kausal für einen bestimmten Schaden ist, wobei gemäß $§ 280$ Abs. 1 BGB das Verschulden des Pflichtverletzers vermutet wird und dieser seinerseits den Entlastungsbeweis gemäß § 280 Abs. 1 S. 2 BGB führen muss.

[28] Zur Überzeugung des Gerichts steht aufgrund der durchgeführten Beweisaufnahme fest, dass der Beklagten unter Berücksichtigung aller tatsächlichen Umstände keine Pflichtverletzung bei der Bewertung und Bewerbung des streitgegenständlichen Kendis vorzuwerfen ist, die kausal für einen Schaden gewesen wäre. Dass im Falle einer ausführlicheren Beschreibung im Katalog - soweit sie der Beklagten möglich gewesen wäre -, einer höheren Schätzwertangabe, der ausdrücklichen Angabe der Provenienz und/oder der richtigen Inbezugnahme auf die Abbildung im Werk von Lunsingh Scheurleer tatsächlich ein höherer Preis erzielt worden wäre, konnte der Kläger nicht beweisen.

[29] Zwischen den Parteien ist entsprechend der Ziffer 1 der Auftragsbedingungen der Beklagten, Anlage K2, Bl. 22 der Akte, ein Kommissionsvertrag gemäß $§ 38$ HGB zustande gekommen. Nach § 384 Abs. 1 HGB ist der Kommissionär verpflichtet, das übernommene Geschäft mit der Sorgfalt eines ordentlichen Kaufmanns auszuführen. Die der Beklagten als Kaufmann obliegende Sorgfalt wurde gewahrt.

\section{[30] 1.}

[31] Nach der Beweisaufnahme steht zur Überzeugung des Gerichts fest, dass der von der Beklagten im Auktionskatalog mit 3.000-4.000 EUR bezifferte Schätzpreis nicht unter Verletzung der obliegenden Sorgfalt zu niedrig angesetzt war. Vielmehr hält der Sachverständige den von der Beklagten angesetzten Schätzpreis zusammengefasst für noch vertretbar, wenn auch etwas knapp. Der Sachverständige Dr. Q erläuterte dazu ausführlich und gut nachvollziehbar, dass er selber den Schätzpreis zwischen 10.000 EUR und 20.000 EUR angesetzt hätte. Es sei jedoch zu berücksichtigen, dass an dem Kendi im Verhältnis zur Abbildung im Lunsingh Scheurleer zwei nicht unwesentliche Teile fehlen. Nach den unwidersprochenen Feststellungen des Sachverständigen ist die Vollständigkeit eines Objekts normalerweise von hoher Relevanz. Das streitgegenständliche Objekt ist nach seinen Ausführungen zwar einzigartig, aber wegen der fehlenden Teile sei der Schätzwert nicht einfach zu ermitteln. Den von Sotheby's für den streitgegenständlichen Kendi festgesetzten Schätzpreis hält der Sachverständige für ungewöhnlich hoch und untypisch. Er sprach im Verlauf der mündlichen Erstattung des Gutachtens von einem „irrsinnigen Preis“. 
[32] Über die streitige Frage, ob der Schätzpreis zwischen der Beklagten und dem Bruder des Klägers abgestimmt wurde, war daher mangels Entscheidungserheblichkeit kein Beweis zu erheben.

[33] Ohnehin ist aufgrund der Ausführungen des Sachverständigen auch nicht davon auszugehen, dass die Angabe eines Schätzwertes in Höhe von 10.000 EUR bis 20.000 EUR zu einem Zuschlag von mehr als den erzielten 75.000 EUR geführt hätte. Der Sachverständige hat bekundet, dass bei Christie's ein ähnliches Wanli-Objekt mit einem Schätzpreis von 2.000 bis 3.000 GBP für 17.300 GBP versteigert wurde. Nach den Ausführungen des Sachverständigen Dr. Q besteht bei einem niedrigen Einstiegspreis zwar die Gefahr, dass dieser nicht übertroffen wird und der Zuschlag zu diesem niedrigen Preis erfolgt. Es sei jedoch auch so, dass das Hochbieten im Saal wichtig ist. Nichts sei tödlicher für eine Auktion, als wenn bei jeder zweiten Sache zu hoch geschätzt wurde und es gar kein Gebot gibt. Vorliegend ist der Schätzwert von 3.000-4.000 EUR mit einem erzielten Preis von 75.000 EUR um ein Vielfaches übertroffen worden. Insbesondere die vom Kläger vorgetragene Vermutung, dass sich bei einem höheren Einstiegspreis weitere Bieter für die Auktion interessiert hätten und ein höherer Zuschlag erzielt worden wäre, lässt sich nach den Ausführungen des Sachverständigen nicht belegen.

\section{[34] 2.}

[35] Dass die Beklagte den Kendi im Auktionskatalog nicht als denjenigen beworben hat, der im Fachbuch Lunsingh Scheurleer beschrieben und abgebildet ist, sondern nur zum Vergleich auf die eigentlich zutreffende Abbildung verwiesen hat, ist ihr nicht als für einen Schaden kausale Pflichtverletzung bei der Bewerbung des streitgegenständlichen Kendis vorzuwerfen. Die Beklagte hat die nach § 384 Abs. 1 HGB ihr als Kommissionär obliegende Sorgfalt eines ordentlichen Kaufmanns beachtet. Maßgebend für die angemessene Sorgfaltsanforderung ist die Art des Geschäfts (Lenz in: Röhricht/Graf von Westphalen/Haas, HGB, 4. Aufl. 2014, § 384 HGB, Rn. 2). Die Beklagte war vorliegend mit der Versteigerung des Kendi im Rahmen eines Kommissionsgeschäfts beauftragt. Anders als in dem Urteil des OLG München (Urteil vom 20.3.2014, Az.: 14 U 764/12) ist die Beklagte nicht bloß ein Varia-Auktionator, sondern ein auf die Versteigerung von Kunstgegenständen spezialisiertes Auktionshaus. Zu ihren Aufgaben gehörte gemäß Ziffer 3. a) der Auftragsbedingungen auch die Katalogisierung und Bewerbung des Gegenstandes. Beim Katalogisieren ging die Beklagte noch davon aus, dass es sich bei dem streitgegenständlichen Kendi nur um ein ähnliches Stück handelt, wie dasjenige, dass in dem Fachbuch Lunsingh Scheurleer abgebildet ist. Aus der Sicht der Kammer sind die von der Beklagten vorgenommenen Anstrengungen zur Korrektur der Angaben im Katalog ausreichend gewesen, um die Sorgfaltsanforderungen eines ordentlichen Kaufmanns zu erfüllen. Noch vor der Versteigerung stellte die Beklagte fest, dass der streitgegenständliche Kendi im Lehrbuch abgebildet ist. Sie hatte jedoch bereits im Katalog zum Vergleich auf die zutreffende Abbildung verwiesen.

[36] Nach dem Ergebnis der Beweisaufnahme steht zur Überzeugung des Gerichts fest, dass die Beklagte, nachdem sie erkannte, dass nicht nur ein ähnlicher Kendi dort abgebildet ist, sondern der streitgegenständliche Kendi nur ohne Deckel und Tülle, sie die Angabe in einer Korrigenda/Addenda-Liste korrigierte und die potentiellen Bieter in der Auktion auf den Fehler hinwies. Der Zeuge Dr. H bekundet, dass die Korrigenda/Addenda-Liste nicht bereits im Katalog, aber schon in der Vorbesichtigung den potenziellen Bietern zugänglich gewesen sei. Auf Nachfrage ergänzte er zudem, dass der Auktionator bei der Versteigerung darauf hingewiesen habe, dass der zu versteigernde Kendi mit dem im Lunsingh Scheurleer abgebildeten Objekt identisch ist. Das Gericht hat keine Zweifel an der Richtigkeit der Einlassung des Zeugen. Zwar liegt bei dem Zeugen Dr. $\mathrm{H}$ als Mitarbeiter der Beklagten ein Interesse am Ausgang des Rechtsstreits nahe. Seine Aussage tätigte er jedoch sachlich und räumte auch Erinnerungslücken ein. Der Zeuge unterfütterte seine Angaben durch die Vorlage des Auktionsprotokolls zu der streitgegenständlichen Versteigerung. Die Schilderungen waren von gleichbleibendem Informationsfluss. Auf Nachfragen des Gerichts oder der Parteien konnte er weitere Details angeben. Widersprüche konnte waren nicht zu erkennen. Auch der Kläger persönliche erklärte in der mündlichen Verhandlung, dass die Ausführungen des Zeugen Dr. $\mathrm{H}$ nicht bestritten werden.

[37] Überdies hat der Kläger nicht dargetan, dass die Abweichung der Angabe im Auktionskatalog zu einem kausalen Schaden geführt hat. Es steht nicht zur Überzeugung des Gerichts fest, dass bei anfänglicher Beschreibung des Kendi als das im Lehrbuch abgebildete Exemplar ein höherer Preis erzielt worden wäre. Nicht zuletzt war auf die zutreffende Abbildung bereits verwiesen worden. Der Sachverständige Dr. Q zeigte insofern auf, dass es sich nicht gerade um Stadtbibliotheksliteratur handele und warf die Frage auf, wer den Hinweisen auf die Literatur folgen möchte und wer es kann.

[38] Daher kann dahinstehen, ob die Beklagte zur Erfüllung der ihr obliegenden Sorgfalt verpflichtet gewesen wäre die Auktion zurückzunehmen, als sie von der fehlerhaften Angabe erfuhr, da nicht dargetan ist, dass in einer späteren Aktion mit richtiger Katalogbeschreibung ein höherer Preis erzielt worden wäre.

\section{[39] 3.}

[40] Auch die Nichtangabe der Provenienz in der Bewerbung durch die Beklagte stellt keine für einen Schaden kausale Pflichtverletzung der Beklagten dar. Gemäß § 384 Abs. 1 HGB hat der Kommissionär das Interesse des Kommittenten wahrzunehmen und dessen Weisungen zu befolgen. Die Weisung muss nicht ausdrücklich erteilt werden, sie kann auch konkludent er- 
gehen (MünchKomm HGB-Häuser, § 384 Rn. 34). Unabhängig von der Frage, ob die Beklagte aufgrund früherer Weisungen davon ausgehen durfte, dass die Provenienz auch bei der streitgegenständlichen Auktion nicht angegeben werden soll, steht nach dem Ergebnis der durchgeführten Beweisaufnahme und unter Berücksichtigung des gesamten Inhalts der mündlichen Verhandlung nicht zur Überzeugung des Gerichts fest, dass sich die Nichtangabe der Provenienz auf das Auktionsergebnis ausgewirkt hat.

[41] Zunächst wies der Sachverständige Dr. Q zutreffend daraufhin, dass in der von der Beklagten in Bezug genommenen Literatur die Provenienz angegeben ist. Er betrachtete die Bekanntgabe der Provenienz durch die Beklagte insofern als nachrangig. Er bekundet zudem eindrücklich, dass die Frage der Kausalität nicht zu beantworten ist, da keine Vergleichsobjekte zur Verfügung stehen. Auch aus der Versteigerung des Kendi durch Sotheby's für 200.000 Britische Pfund, lässt sich kein Rückschluss auf diese Frage ziehen. Der Kläger hat nicht dargetan, dass die Angabe der Provenienz für den Londoner Bieter zur Abgabe des hohen Gebots ausschlaggebend war. Zudem hat der Sachverständige darauf hingewiesen, dass London und New York ein anderer Markt ist.

\section{[42] II.}

[43] Da die Klage in der Hauptsache keinen Erfolg hat, kann der Kläger auch nicht mit dem geltend gemachten Nebenanspruch auf Erstattung der außergerichtlichen Anwaltskosten aus Antrag zu 2) durchdringen.

\section{[44] III.}

[45] Der nicht nachgelassene Schriftsatz der Beklagten vom 11.9.2017 veranlasst nicht die Wiedereröffnung der mündlichen Verhandlung. Er enthält keinen für die Entscheidung erheblichen Vortrag. 